\title{
Scale and Scaling in the Climate System
}

\author{
Shaun Lovejoy ${ }^{1}$, M. Crucifix ${ }^{2}$ and A. de Vernal ${ }^{3}$
}

Montreal, Canada, 5-7 October 2015

Forty years ago, at the dawn of the revolution in paleoclimate data, it was believed that atmospheric variability essentially consisted of an uninteresting, spectrally flat (white noise) "background", interspersed with periodic and quasi-periodic processes such as the diurnal and annual cycles, the Southern Oscillation and Milankovitch cycles (Fig. 1, bottom). Since then, instrumental and paleo data have shown this picture is wrong by a large factor (Fig. 1, top): the background displays nontrivial "variability across scales" that can be roughly divided into five power law ("scaling") regimes. This state of affairs, underscoring the need for a fresh look at climate variability and theory, motivated this workshop.

The first objective was to get nonlinear geoscientists and paleoclimate scientists to think about effective ways to characterize climate fluctuations, understand their causes, and focus on these aspects when analyzing paleoclimate data; the second was to develop a common research agenda.

The workshop successfully brought communities together with expertise in recent climate (11 participants), paleoclimate (21), climate modeling (9), and nonlinear, statistical and stochastic mathematics (22). Participants, including 14 students, came from Canada, USA, Europe, Asia, and Latin America.

The ice core and marine paleoclimate experts in particular provided an authoritative account of what paleoclimate data can offer (E. Wolff, A. De Vernal), including various pitfalls (M. Kucera). The more applied-mathematics-inclined speakers discussed the origin of long-memory process (C. Franzke), their detection (K. Rypdal), and some difficulties (T. Nielsen). A wide range of timescales was covered from the instrumental periods permitting the study of spatio-temporal variability (H. Frederiksen). We now have enough accurate data to address questions that were previously impossible, such as the gain between forcing and response at timescales of hundreds of thousand years ( $P$. Huybers). Specific physical mechanisms associated with the carbon budget (K. Kohfeld) or atmosphere dynamics at the Last Glacial Maximum (W. Roberts) were discussed. Finally, the value of a good knowledge of long-memory processes was emphasized for seasonal forecasts (R. Hébert), extreme events (S. Innocenti) or global warming (S. Lovejoy).

A number of common research themes emerged around the variability of the climate, especially at centennial to multi-millennial scales. For example, paleoclimate scientists tend to refer to modes of changes called "NAO-like" or "El-Niño like", but how does it actually connect to the interannual modes of variability described by meteorologists? This variability must also be understood from a physical point of view and include the implications for climate change predictions and projections.

The overall result was the creation of a PAGES working group "Climate Variability Across Scales" (CVAS, see Program News article on page 32 ).

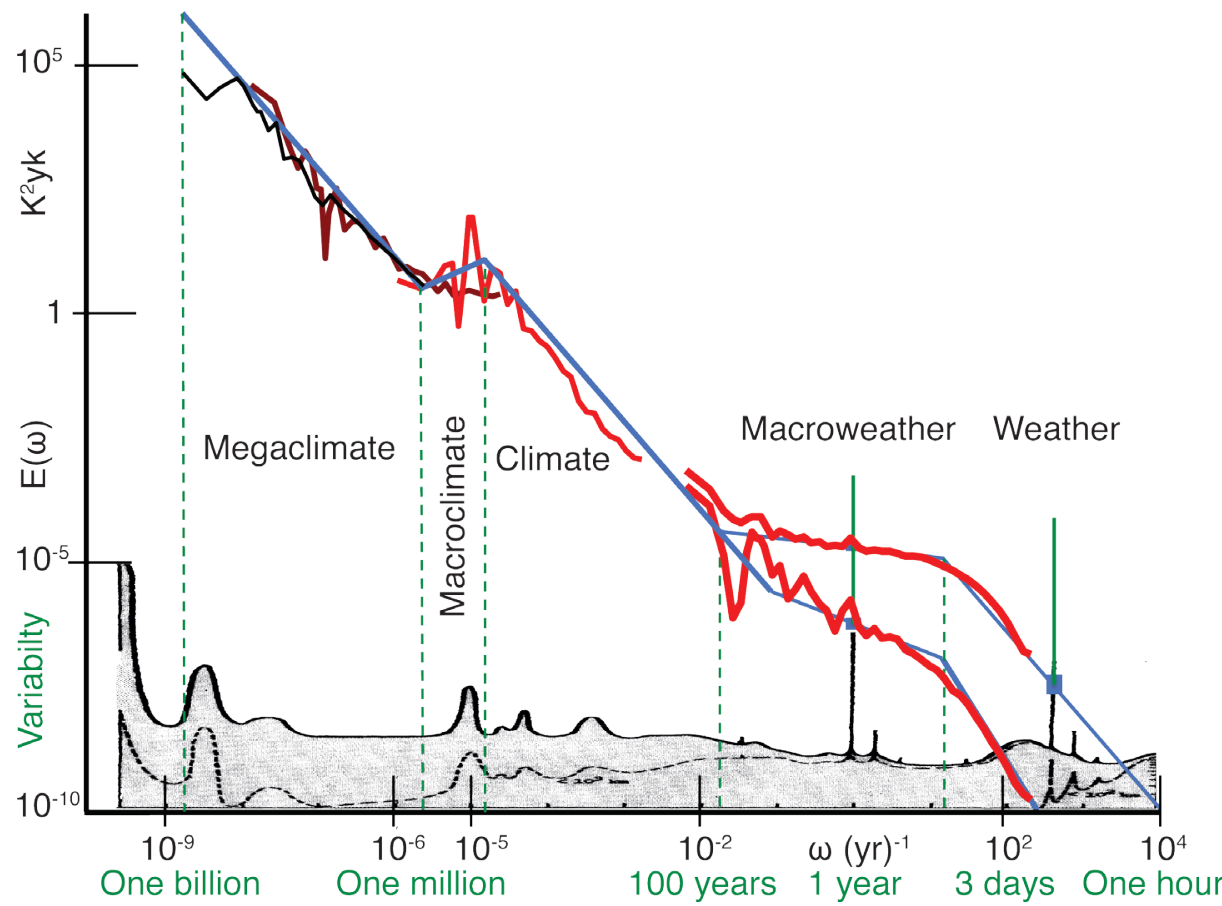

Figure 1: The temperature spectrum $(E(\omega))$ giving the variance per interval of frequency $(\omega)$. The bottom (grey) is M. Mitchell's "educated guess" showing the still dominant view of a fairly flat (white noise) "background" interspersed with spikes corresponding to important (quasi-periodic) processes (Mitchell 1976). Top curves are based on instrumental and paleo-data and their range differs from Mitchell's guess by a factor of roughly $10^{15}$. In actual fact, the "background" accounts for nearly all the variance, so that the roles of foreground and background must be inverted: Mitchell must be "stood on his head". To a first approximation, the spectrum can be divided into five power law regimes (linear on this log-log plot, blue reference lines). At the far right, there are two nearly parallel instrumental curves corresponding to the spectra of temperatures averaged over the globe (bottom) and over $5^{\circ} \times 5^{\circ}$ (top). Also shown (green) are the spikes corresponding to diurnal and annual cycles. Adapted from Lovejoy (2015), which has the full details of the data sources. 